Quim. Nova, Vol. 35, No. 9, 1858-1864, 2012

\title{
AVALIAÇÃO DE MÉTODOS DE EXTRAÇÃO PARA A DETERMINAÇÃO DE CROMO E NÍQUEL EM FORMULAÇÕES FARMACÊUTICAS E EM MATÉRIAS-PRIMAS USADAS NA FABRICAÇÃO DE MEDICAMENTOS À BASE DE CEFALEXINA E CIPROFLOXACINO
}

\author{
Carlos Eduardo R. de Paula \\ Departamento de Química Analítica, Universidade Federal Fluminense, Outeiro de São João Batista, s/n, 24020-141 Niterói - RJ / \\ Departamento de Química, Universidade Federal Rural do Rio de Janeiro, BR-465, km 7, 23890-000 Seropédica - RJ, Brasil \\ Daniel M. Brum, Luiz Fernando S. Caldas e Ricardo J. Cassella* \\ Departamento de Química Analítica, Universidade Federal Fluminense, Outeiro de São João Batista, s/n, 24020-141 Niterói - RJ, \\ Brasil
}

Recebido em 31/1/12; aceito em 25/5/12; publicado na web em 10/8/12

\begin{abstract}
EVALUATION OF EXTRACTION METHODS FOR THE DETERMINATION OF Cr AND Ni IN PHARMACEUTICAL FORMULATIONS AND RAW MATERIALS USED IN THE PRODUCTION OF CEPHALEXIN AND CIPROFLOXACIN MEDICINES. This paper reports the evaluation of extraction strategies for the treatment of medicine samples to determine chromium and nickel by GFAAS. Different approaches for extraction were evaluated and the most efficient involved magnetic stirring. The metals were quantitatively extracted by stirring $0.20 \mathrm{~g}$ samples with $25 \mathrm{~mL}$ of $2.0 \mathrm{~mol} \mathrm{~L}^{-1} \mathrm{HCl}$ solution for $60 \mathrm{~min}$. The developed method was successfully applied for the determination of $\mathrm{Cr}$ and $\mathrm{Ni}$ in tablets containing antibiotics and raw materials, with cephalexin and ciprofloxacin as active ingredients.
\end{abstract}

Keywords: extraction procedures; medicines; graphite furnace atomic absorption spectrometry.

\section{INTRODUÇÃO}

O controle de qualidade de matérias-primas de uso farmacêutico e de formulações farmacêuticas é uma etapa de grande importância na produção de medicamentos e abrange a amostragem, a verificação das especificações, os ensaios de controle de qualidade, os procedimentos de organização e a geração de documentação de liberação, que asseguram que os ensaios necessários e relevantes foram executados. A Farmacopeia Brasileira, $5^{\text {a }}$ ed. (2010), ${ }^{1}$ enfatiza os três tipos de ensaios que devem ser realizados para garantir a qualidade dos produtos farmacêuticos (e suas matérias-primas): identificação, doseamento e pureza. Entre os contaminantes inorgânicos mais comuns destacam-se os metais pesados, o cloreto e o sulfato. ${ }^{2}$

A contaminação por metais pesados em fármacos normalmente está associada à sua presença na matéria-prima e/ou ao próprio processo de produção do medicamento, visto que os equipamentos utilizados na produção são constituídos, na sua maioria, de aço inoxidável. Este é composto de aproximadamente 10\% de níquel, 18\% de cromo e $70 \%$ de ferro. Os principais fatores que podem afetar a migração destes elementos para os produtos finais são o $\mathrm{pH}$, o tempo de exposição e a temperatura. ${ }^{3}$ Assim, durante o processo de produção, quantidades significativas de $\mathrm{Ni}$ e $\mathrm{Cr}$ podem ser transferidas para os medicamentos e estes teores devem ser controlados devido à toxicidade destes metais.

A USP (The United States Pharmacopeia) ainda propõe a utilização de um ensaio limite, semiquantitativo, para a determinação de metais em medicamentos, utilizando a reação entre os metais pesados e o íon sulfeto. ${ }^{4}$ Neste teste, o resultado é expresso como o somatório das concentrações dos metais pesados contaminantes na amostra, que não deve ultrapassar o limite de $20 \mu \mathrm{g} \mathrm{g}^{-1} .5$ Os ensaios não seletivos vêm sendo paulatinamente substituídos por determinações específicas envolvendo instrumentação analítica baseada em técnicas, tais como, a espectrometria de absorção atômica com chama

*e-mail: cassella@vm.uff.br
(FAAS), ${ }^{6,7}$ a espectrometria de absorção atômica com forno de grafite (GFAAS), ${ }^{8,9}$ a espectrometria de emissão ótica em plasma de argônio acoplado por indução (ICP-OES) ${ }^{10,11}$ e a espectrometria de massas com fonte de plasma acoplado por indução (ICP-MS). ${ }^{12,13}$ Com isto, busca-se o desenvolvimento de métodos que forneçam informações de modo mais veloz e específico, a fim de atender às necessidades das indústrias farmacêuticas.

Por sua vez, a aplicação das técnicas espectrométricas descritas acima requer, na grande maioria dos casos, um pré-tratamento da amostra, a fim de convertê-la em uma forma adequada para introdução nos equipamentos analíticos. A etapa de pré-tratamento da amostra pode ser considerada a etapa crítica do processo analítico, uma vez que requer um gasto de tempo elevado e está sujeita a problemas, tais como, a contaminação das amostras e perdas do analito durante a manipulação das amostras. ${ }^{14,15}$

No caso de amostras sólidas, diferentes procedimentos têm sido adotados para a sua conversão em uma forma líquida, apropriada para injeção nos instrumentos. Indubitavelmente, a digestão ácida, seja por aquecimento convencional ou utilizando-se radiação micro-ondas, é o mais popular dos métodos de dissolução de amostras sólidas. O problema é que estes procedimentos, além de laboriosos, são lentos (principalmente quando o aquecimento convencional é utilizado) e exigem o manuseio de reagentes tóxicos e corrosivos. A digestão de amostras sólidas também pode ser levada a cabo por via seca, seja através do aquecimento direto das amostras ou na presença de fundentes. ${ }^{16,17}$ Ainda, métodos alternativos podem ser utilizados, tais como, a piro-hidrólise ${ }^{18}$ ou a combustão induzida por radiação micro-ondas. ${ }^{19}$ Em muitos casos, a lixiviação dos analitos presentes nas amostras pode ser uma alternativa interessante aos métodos que empregam a destruição (ou dissolução) total da matriz, uma vez que os processos de extração são, em geral, mais rápidos e utilizam soluções diluídas de ácidos..$^{20,21}$

O objetivo desse trabalho foi avaliar a possibilidade de utilização de diferentes metodologias de extração para a determinação de $\mathrm{Cr}$ e Ni por GFAAS em amostras de formulações farmacêuticas 
e matérias-primas de uso farmacêutico contendo os antibióticos cefalexina e ciprofloxacino. As metodologias de extração testadas envolveram a extração empregando agitação magnética, a extração assistida por banho de ultrassom e a extração assistida por radiação micro-ondas focalizada.

\section{PARTE EXPERIMENTAL}

\section{Instrumentação}

Foi empregado um espectrômetro de absorção atômica com atomização eletrotérmica em forno de grafite Varian (Mulgrave, Austrália), modelo AA240Z, equipado com amostrador automático Varian PSD 120, forno de grafite GTA 120 e corretor de fundo por efeito Zeeman. A atomização foi realizada a partir da parede de tubos de grafite eletrolítico recobertos com grafite pirolítico, também fornecidos pela Varian.

Lâmpadas de catodo oco monoelementar de $\mathrm{Cr}$ e Ni foram empregadas como fontes de radiação e operadas com correntes de 7 e 4 $\mathrm{mA}$, respectivamente. A medição de $\mathrm{Cr}$ foi efetuada em 357,9 nm e a de $\mathrm{Ni}$ em 232,0 nm. Em ambos os casos, a resolução espectral foi de $0,2 \mathrm{~nm}$. Todas as medições foram baseadas em valores de absorbância integrada e o gás de proteção utilizado foi o argônio (99,99\%), fornecido pela Linde Gases (Macaé, Brasil).

Também foram utilizados, nos experimentos de extração, um agitador magnético Fisatom (São Paulo, Brasil), modelo 752-A; um banho de ultrassom Unique (São Paulo, Brasil), modelo 1600, com frequência constante de $40 \mathrm{MHz}$ e um forno de micro-ondas com radiação focalizada CEM (Matthews, EUA), modelo Star 2, com potência nominal de $950 \pm 50 \mathrm{~W}$. Tubos e condensadores de quartzo foram usados para inserir as amostras no forno de micro-ondas.

\section{Reagentes e soluções}

Todas as soluções foram preparadas empregando-se água produzida em um sistema Direct-Q 3, fornecido pela Millipore (Milford, EUA). A água utilizada apresentou resistividade sempre igual ou superior a $18,2 \mathrm{M} \Omega \mathrm{cm}$.

As soluções analíticas de cromo e níquel utilizadas foram preparadas em balões volumétricos de $5 \mathrm{~mL}$, a partir da diluição adequada das soluções estoque de $1000 \mathrm{mg} \mathrm{L}^{-1}$ fornecidas pela Tedia (Fairfield, $\mathrm{OH}, \mathrm{EUA})$. O diluente empregado foi sempre compatível com a solução utilizada nas extrações.

As soluções de $\mathrm{HCl}$ e $\mathrm{HNO}_{3}$ utilizadas foram preparadas a partir da diluição adequada dos ácidos concentrados (grau metais traço) fornecidos pela Tedia, com água.

A solução oxidante utilizada na dissolução total das amostras foi preparada pela mistura de $300 \mathrm{~mL}$ de $\mathrm{HCl}$ concentrado com $100 \mathrm{~mL}$ de $\mathrm{HNO}_{3}$ concentrado, em banho de gelo. Os ácidos concentrados (grau metais traço) utilizados também foram fornecidos pela Tedia.

\section{Procedimento geral utilizado nas extrações}

As amostras utilizadas, tanto na forma farmacêutica quanto na forma pura, foram trituradas em gral e pistilo de porcelana com o intuito de diminuir o tamanho das partículas e assegurar uma maior homogeneidade das mesmas. A fim de obter amostras representativas, 20 comprimidos de cada amostra foram triturados conjuntamente, no caso das formulações farmacêuticas. As amostras de matérias-primas já foram recebidas na forma pulverizada, sendo apenas trituradas mais uma vez para garantir a sua homogeneidade. Uma massa definida de amostra triturada foi pesada diretamente no frasco empregado na extração (tubo de polietileno de $50 \mathrm{~mL}$ para a agitação magnética e extração assistida por banho de ultrassom e frasco de quartzo para a extração assistida por radiação micro-ondas focalizada), $25 \mathrm{~mL}$ da solução extratora adequada foram então adicionados e o procedimento foi levado a cabo de acordo com as condições estabelecidas em cada experimento de otimização. Ao final da extração, as suspensões obtidas em cada processo foram filtradas em filtro de membrana de acetato de celulose de $0,22 \mu \mathrm{m}$ de diâmetro de poro e $30 \mathrm{~mm}$ de diâmetro com o auxilio de uma seringa. Quando necessário, o extrato obtido foi diluído com água antes da medição no espectrômetro de absorção atômica.

\section{Dissolução total assistida por radiação micro-ondas focalizada}

Na dissolução total assistida por radiação micro-ondas focalizada, alíquotas de 0,20 g das amostras pulverizadas foram pesadas diretamente no frasco do forno de micro-ondas e, em seguida, foram adicionados $20 \mathrm{~mL}$ da solução oxidante. $\mathrm{O}$ frasco foi então introduzido no equipamento e foi executado o programa de aquecimento mostrado na Tabela 1. Ao final do programa de aquecimento, os frascos foram resfriados à temperatura ambiente por aproximadamente 15 min. A solução restante foi transferida para um frasco de polietileno e o volume foi completado até $25 \mathrm{~mL}$ com água. Quando necessário, as soluções obtidas foram filtradas em filtro de membrana e diluídas com água.

Tabela 1. Programa de aquecimento para extração assistida por micro-ondas focalizada e dissolução total da amostra

\begin{tabular}{ccccc}
\hline \multirow{2}{*}{ Etapa } & \multicolumn{2}{c}{$\begin{array}{c}\text { Extração assistida por } \\
\text { micro-ondas focalizada }\end{array}$} & \multicolumn{2}{c}{ Dissolução total } \\
\cline { 2 - 5 } & $\begin{array}{c}\text { Tempo } \\
(\mathrm{min})\end{array}$ & $\begin{array}{c}\text { Temperatura } \\
\left({ }^{\circ} \mathrm{C}\right)\end{array}$ & $\begin{array}{c}\text { Tempo } \\
(\mathrm{min})\end{array}$ & $\begin{array}{c}\text { Temperatura } \\
\left({ }^{\circ} \mathrm{C}\right)\end{array}$ \\
\hline (1) Rampa & 0,5 & 70 & 3 & 90 \\
Patamar & 15 & 70 & 10 & 90 \\
$(2)$ Rampa & $*$ & $*$ & 1 & 110 \\
Patamar & $*$ & $*$ & 15 & 110 \\
\hline
\end{tabular}

\section{Determinação de cromo e níquel em amostras de cefalexina e ciprofloxacino}

As determinações de $\mathrm{Cr}$ e $\mathrm{Ni}$ nos extratos obtidos nas extrações foram realizadas por GFAAS, utilizando os parâmetros instrumentais apresentados nos itens anteriores e a programação de temperatura apresentada na Tabela 2.

Tabela 2. Programas de temperatura empregados na determinação de cromo e níquel por GFAAS em amostra de cefalexina e ciprofloxacino

\begin{tabular}{|c|c|c|c|c|}
\hline Etapas & $\begin{array}{c}\text { Temperatura } \\
\left({ }^{\circ} \mathrm{C}\right)\end{array}$ & Rampa (s) & Patamar (s) & $\begin{array}{l}\text { Vazão de Ar } \\
\left.(\mathrm{mL} \mathrm{min})^{-1}\right)\end{array}$ \\
\hline \multirow[t]{3}{*}{ Secagem } & 85 & 5 & 0 & 300 \\
\hline & 95 & 40 & 0 & 300 \\
\hline & 120 & 10 & 0 & 300 \\
\hline Pirólise & $\begin{array}{c}1000(\mathrm{Cr}) ; 800 \\
(\mathrm{Ni})\end{array}$ & 2 & 6 & 300 \\
\hline Atomização & $\begin{array}{l}2500(\mathrm{Cr}) \\
2500(\mathrm{Ni})\end{array}$ & 0,9 & 2,1 & 0 \\
\hline Limpeza & $\begin{array}{l}2600(\mathrm{Cr}) \\
2600(\mathrm{Ni})\end{array}$ & 2,0 & 0 & 300 \\
\hline
\end{tabular}

Em todas as medições, $20 \mu \mathrm{L}$ de solução foram diretamente injetados dentro do tubo de grafite e, em seguida, foi iniciado o programa de temperatura. A atomização dos analitos foi efetuada a partir das paredes dos tubos de grafite, que eram compostos de 
grafite eletrolítico recoberto com grafite pirolítico. Não foi empregado qualquer modificador químico, uma vez que não foi verificada a necessidade de estabilizar os analitos dentro do tubo de grafite.

Em todos os experimentos a determinação dos analitos foi realizada em triplicata e foi efetuado um rigoroso controle dos brancos, para evitar problemas de contaminação.

\section{Descontaminação do material utilizado}

Toda vidraria e frascos plásticos utilizados foram lavados com água corrente, em seguida enxaguados com água desionizada e imersos em solução $10 \%$ v/v de ácido clorídrico por 24 h. Após este tempo, os materiais foram cuidadosamente lavados com água desionizada e secos em estufa a $40{ }^{\circ} \mathrm{C}$ (exceto material volumétrico), evitando qualquer contato com materiais metálicos. Em seguida foram armazenados, até o seu uso, em um ambiente fechado isento de poeira.

\section{RESULTADOS E DISCUSSÃO}

Os resultados obtidos são apresentados em três partes: a primeira trata da otimização do programa de temperatura para determinação de cromo e níquel utilizando a técnica GFAAS, representada pela construção das curvas de pirólise e atomização; a segunda, da otimização dos parâmetros para extração de cromo e níquel em matérias-primas de uso farmacêutico e em formulações farmacêuticas e, a terceira mostra os parâmetros analíticos dos métodos e a sua aplicação em amostras reais.

\section{Otimização do programa de temperatura para determinação de $\mathrm{Cr}$ e Ni por GFAAS}

Na determinação de metais por espectrometria de absorção atômica em forno de grafite, a otimização do programa de aquecimento é uma das etapas mais importantes, uma vez que as temperaturas a que a amostra é submetida afetam consideravelmente a sensibilidade da metodologia.

A otimização do programa de temperatura do cromo e níquel em solução aquosa e em meio da amostra foi realizada analisando-se as curvas de temperatura de pirólise e atomização. Após a otimização do programa de temperatura foi possível determinar a temperatura máxima permitida na etapa de pirólise sem que ocorra perda do analito, bem como a melhor temperatura de atomização.

As curvas de pirólise e de atomização em solução aquosa foram construídas a partir dos sinais de absorbância integrada obtidos com uma solução padrão contendo $6 \mu \mathrm{g} \mathrm{L}^{-1}$ de $\mathrm{Cr}$ e $10 \mu \mathrm{g} \mathrm{L}^{-1}$ de Ni. No caso das curvas de pirólise e atomização em meio da amostra, foram utilizados os sinais gerados a partir da medição de Cr e Ni no extrato obtido (com agitador magnético) a partir de 0,20 g de uma amostra com $25 \mathrm{~mL}$ de $\mathrm{HCl} \mathrm{1,0} \mathrm{mol} \mathrm{L}{ }^{-1}$, durante $90 \mathrm{~min}$. A Figura 1 mostra as curvas de pirólise e de atomização para os analitos em solução aquosa e em meio da amostra. Os perfis das curvas de pirólise e atomização para as espécies de interesse foram muito semelhantes, tanto em meio aquoso quanto em meio da amostra. Isto indica que não deve haver interferência de qualquer componente presente na amostra, que porventura seja extraído, sobre o comportamento térmico dos analitos dentro do tubo de grafite. As diferenças observadas entre as intensidades dos sinais obtidos devem ser, então, prioritariamente devidas às diferenças entre as concentrações de $\mathrm{Cr}$ e $\mathrm{Ni}$ nas soluções padrão e no extrato da amostra. Vale ressaltar que os valores de absorção de fundo encontrados estiveram sempre dentro da faixa de trabalho do equipamento e puderam ser perfeitamente corrigidos.

Com base nos dados obtidos, as temperaturas de pirólise escolhidas para o método foram 1000 e $800^{\circ} \mathrm{C}$ para $\mathrm{Cr}$ e Ni, respectivamente.
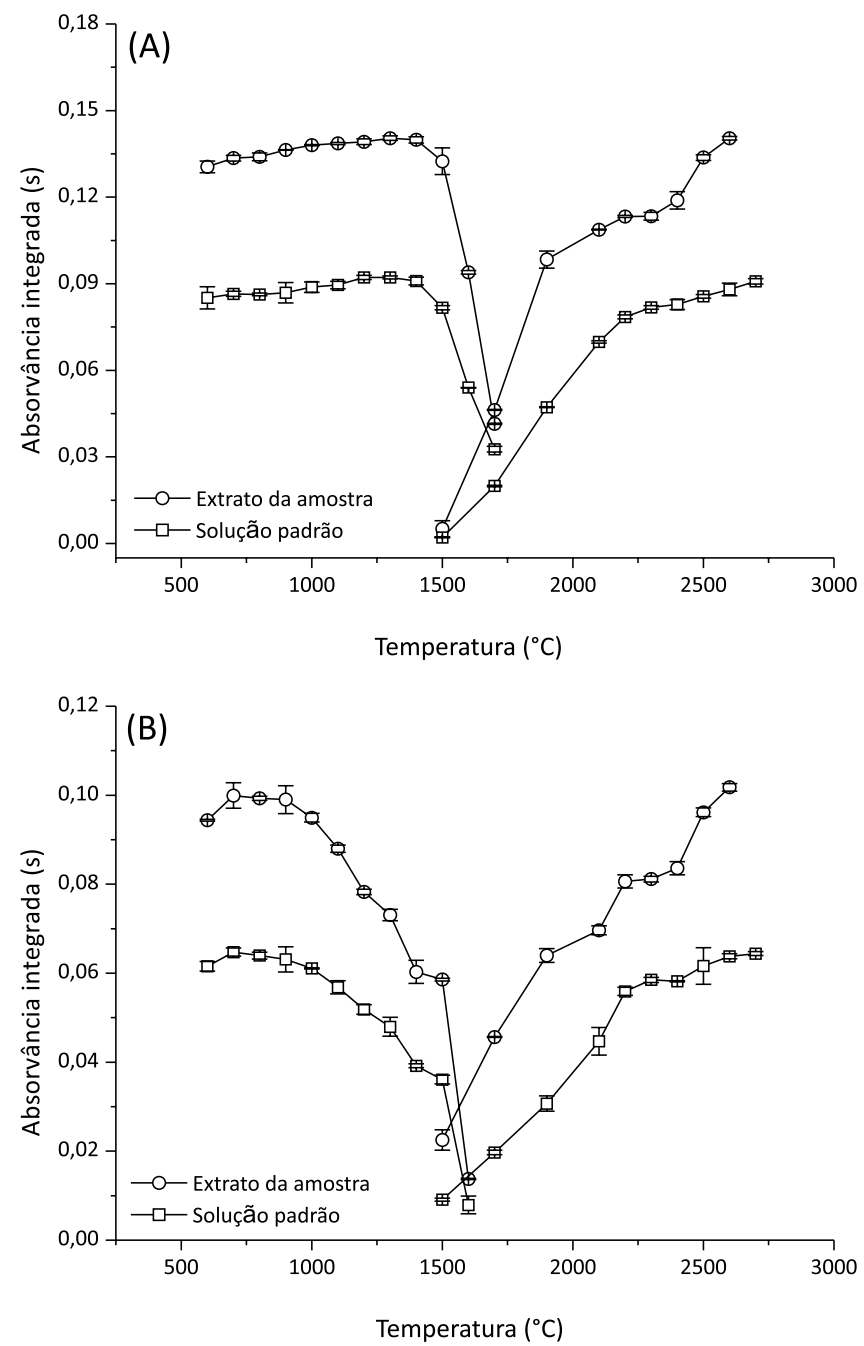

Figura 1. Curvas de pirólise e atomização para cromo (A) e níquel (B) em solução padrão e meio do extrato da amostra

Já as temperaturas de atomização foram de $2500^{\circ} \mathrm{C}$ tanto para o $\mathrm{Cr}$ quanto para o $\mathrm{Ni}$.

\section{Otimização da extração de cromo e níquel}

O processo de extração foi estudado utilizando-se apenas uma amostra como referência. Para tal, as concentrações de $\mathrm{Cr}$ e Ni foram determinadas empregando-se a dissolução total da amostra em forno de micro-ondas com radiação focalizada e a medição por GFAAS. As eficiências de extração obtidas em cada experimento foram calculadas tomando-se como referência as concentrações totais de $\mathrm{Cr}$ e Ni encontradas, que foram iguais a 1,01 e $0,91 \mu \mathrm{g} \mathrm{g}^{-1}$, respectivamente. Diferentes modos de extração (agitação magnética, extração assistida por ultrassom e extração assistida por radiação micro-ondas focalizada) foram inicialmente avaliados com o intuito de comparar suas eficiências.

A otimização foi efetuada de modo univariado, estudando-se sistematicamente a influência de diferentes parâmetros sobre os processos de extração, tais como, a natureza do ácido, a concentração do ácido, o tempo de extração e a influência da massa de amostra.

\section{Efeito da concentração e da natureza do ácido empregado na solução extratora}

A concentração do ácido é essencial no processo de extração 
dos metais, uma vez que o íon $\mathrm{H}^{+}$deve ser responsável pelo deslocamento dos íons metálicos presentes na matriz sólida da amostra. Adicionalmente, a natureza do ácido empregado também pode exercer um papel importante, uma vez que o seu poder oxidante pode auxiliar na oxidação da amostra, liberando mais facilmente os íons metálicos para a solução. Neste estudo, os efeitos da natureza do ácido e da concentração do ácido foram avaliados simultaneamente utilizando-se soluções diluídas de ácido clorídrico, ácido nítrico e uma mistura de ambos os ácidos.

Nas extrações assistidas por agitação magnética e por ultrassom, a influência da concentração do ácido foi avaliada variando-se as concentrações dos ácidos (no caso da mistura, concentrações equivalentes de $\mathrm{HCl}$ e $\mathrm{HNO}_{3}$ foram empregadas de modo que a concentração total fosse igual aos valores estipulados) de 0,1 a 2,0 $\mathrm{mol} \mathrm{L}^{-1}$. O tempo de extração foi fixado em 60 min e a extração foi realizada com $0,20 \mathrm{~g}$ de amostra e $25 \mathrm{~mL}$ de solução extratora. Na extração assistida por micro-ondas focalizada, a verificação da influência da concentração e da natureza do ácido foi realizada empregando-se as mesmas soluções extratoras, a mesma massa de amostra e o mesmo volume de solução. Entretanto, o tempo de extração foi fixado em 15 min e a temperatura de extração foi estabelecida em $70{ }^{\circ} \mathrm{C}$. É importante salientar que estas condições de extração foram muito mais brandas do que aquelas empregadas na dissolução total da amostra, resultando em uma economia de tempo e reagentes.

De modo geral, soluções contendo apenas $\mathrm{HCl}$ apresentaram maior eficiência de extração, especialmente para soluções com concentrações de ácido iguais ou superiores a $0,5 \mathrm{~mol} \mathrm{~L}^{-1}$. Este comportamento indica que o poder oxidante da mistura parece não exercer um efeito preponderante sobre o processo de extração, sendo este dependente, em maior extensão, da concentração de íons $\mathrm{H}^{+} \mathrm{e}$, principalmente, do efeito do ânion. A presença do cloreto (oriundo da dissociação do $\mathrm{HCl}$ ) parece auxiliar na extração, possivelmente devido ao seu mais alto poder de complexação em relação ao nitrato.

Como esperado, o aumento da concentração do ácido na solução extratora resultou em um aumento da eficiência de extração, certamente devido ao aumento da concentração, no meio extrator, das espécies que participam do processo da extração, $\mathrm{H}^{+}$e cloreto.

A eficiência de extração também se mostrou dependente da estratégia de extração empregada. Extrações quantitativas de ambos os metais foram obtidas somente com o uso da agitação magnética (Figura 2). Tanto o emprego da extração assistida por ultrassom quanto da extração assistida por radiação micro-ondas focalizada resultaram em extrações incompletas, sendo obtidas eficiências de extração máximas de 55 e $85 \%$, respectivamente (Figuras $1 \mathrm{~S}$ e $2 \mathrm{~S}$, material suplementar). Estes resultados evidenciam que o aumento do contato entre amostra e solução extratora é fundamental para o processo de lixiviação dos metais, uma vez que as extrações com o banho de ultrassom e com o forno de micro-ondas não permitem uma agitação eficiente da mistura.

\section{Efeito do tempo de extração}

Como visto anteriormente, foram observadas diferenças significativas entre as eficiências de extração quando diferentes estratégias de extração foram empregadas. Apesar das diferenças terem sido creditadas às diferenças entre as eficiências de agitação, poderiam estar também relacionadas ao tempo de contato entre a amostra e a solução extratora, especialmente para o caso da extração assistida por radiação micro-ondas, em que foi utilizado um menor tempo de irradiação (15 min). Assim, realizou-se outro experimento variando-se o tempo de extração entre 1 e 90 min para cada sistema. A fim de tentar alcançar extrações quantitativas em menores tempos de extração, foram empregadas as melhores condições (em termos de
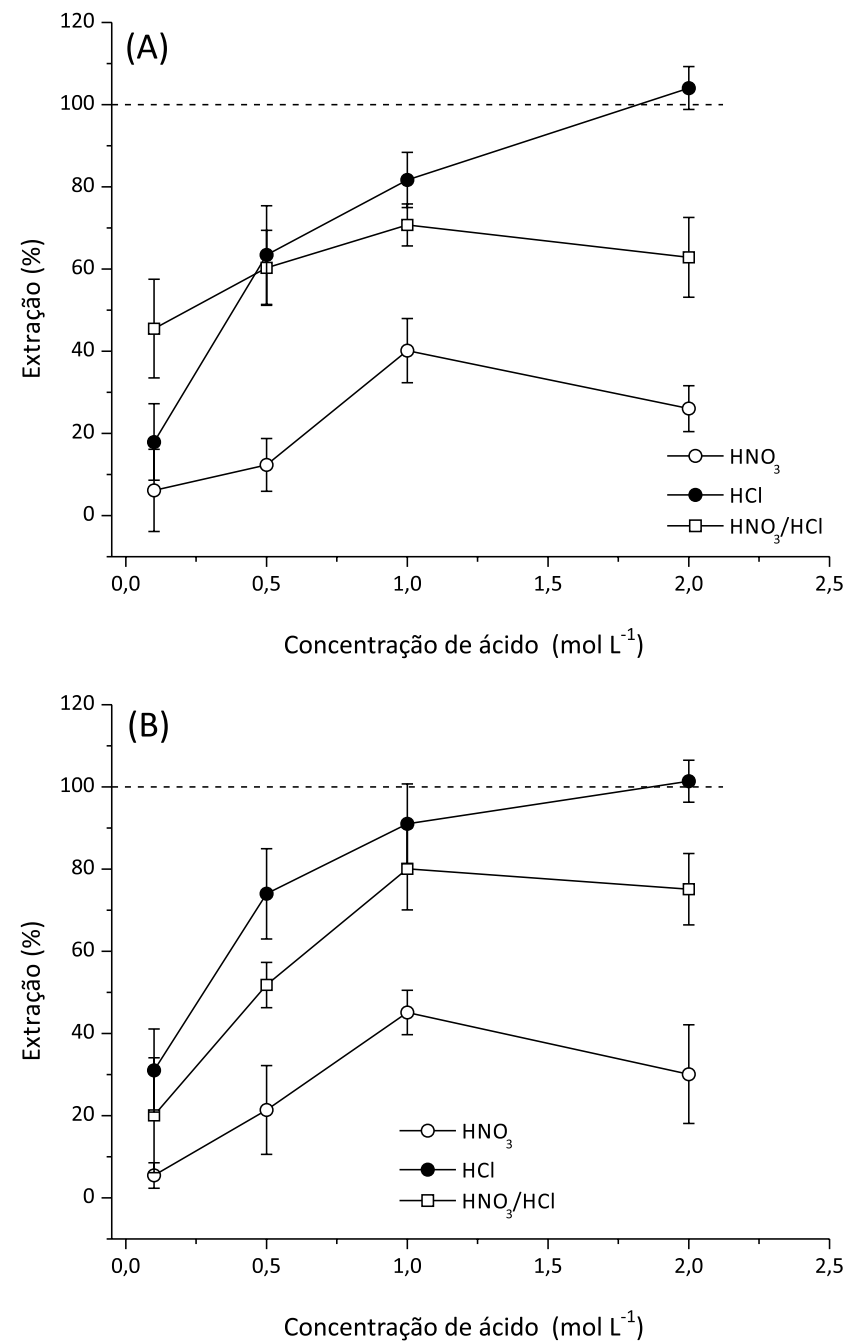

Figura 2. Influência da natureza e da concentração do ácido na extração de $\mathrm{Cr}$ (A) e Ni (B) por agitação magnética. Detalhes sobre os tempos de extração podem ser encontrados no texto

concentração de ácido) para cada caso (natureza da solução extratora). A massa de amostra empregada foi de $0,20 \mathrm{~g}$ e o volume de solução extratora foi de $25 \mathrm{~mL}$. Para a extração assistida por micro-ondas focalizada a temperatura de extração foi mantida em $70{ }^{\circ} \mathrm{C}$, como nos experimentos anteriores.

Novamente, foram obtidas extrações quantitativas para $\mathrm{Cr}$ e Ni somente no caso do uso da agitação magnética (Figura 3). Para este modo de extração, o tempo apresentou efeito significativo sobre a extração de $\mathrm{Cr}$ e Ni na amostra de referência, principalmente no caso em que uma solução de $\mathrm{HCl} 2 \mathrm{~mol} \mathrm{~L}^{-1}$ foi utilizada na extração, sendo observado um aumento da eficiência de extração com o aumento do tempo de extração até 60 min. Após este tempo, as eficiências de extração permaneceram constantes, não havendo variação significativa das quantidades dos metais extraídos. O emprego de soluções de $\mathrm{HNO}_{3}$ e $\mathrm{HNO}_{3} / \mathrm{HCl} 1 \mathrm{~mol} \mathrm{~L}^{-1}$ não resultou em extrações quantitativas de $\mathrm{Cr}$ e Ni, mesmo com o aumento dos tempos de extração até 90 min.

As extrações assistidas por ultrassom e por radiação micro-ondas focalizada também não permitiram a extração quantitativa dos analitos, mesmo quando foram empregados tempos de extração de 90 min (Figuras $3 \mathrm{~S}$ e 4S, material suplementar). Os percentuais de extração máximos obtidos ficaram em patamares semelhantes aos do experimento anterior, ou seja, em torno de $60 \%$ para extração assistida por ultrassom e $80 \%$ para extração assistida por radiação micro-ondas focalizada. 

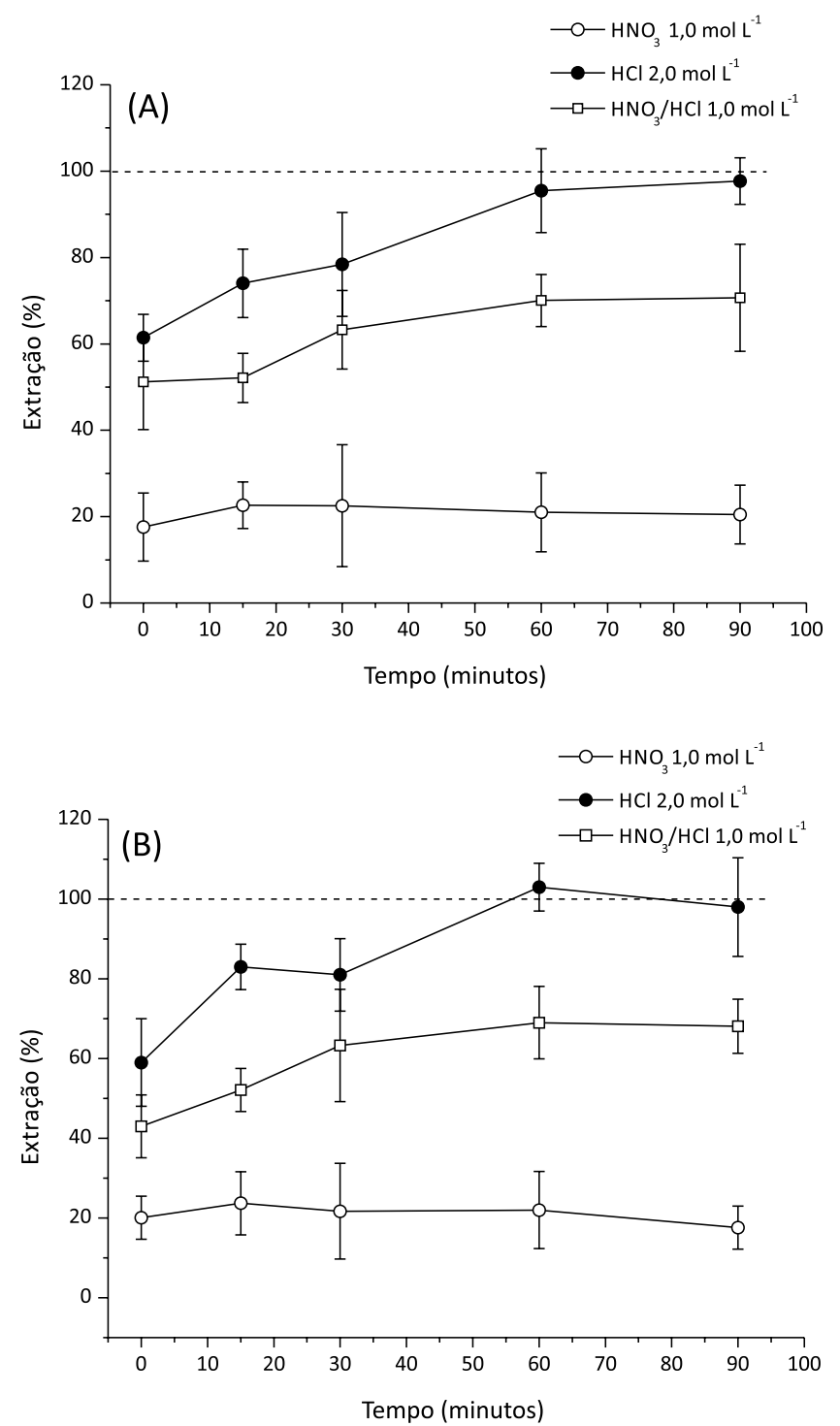

Figura 3. Influência do tempo na extração de $\mathrm{Cr}(\mathrm{A})$ e $\mathrm{Ni}$ (B) por agitação magnética. Detalhes sobre a concentração de ácido (ou mistura) na solução extratora podem ser encontrados no texto

Os resultados obtidos indicaram que maiores eficiências de extração, próximas a $100 \%$, tanto para $\mathrm{Cr}$ como para $\mathrm{Ni}$, podem ser obtidas com a agitação da amostra $(0,20 \mathrm{~g})$ com uma solução de $\mathrm{HCl} 2 \mathrm{~mol}$ $\mathrm{L}^{-1}(25 \mathrm{~mL})$ por 60 min em um sistema de agitação magnética. Estas condições foram então selecionadas para os experimentos posteriores.

\section{Influência da relação massa de amostra/volume de solução extratora}

Um aspecto relevante nos procedimentos de preparação de amostras é a quantidade de amostra (massa) que pode ser processada. Esta variável afeta diretamente a sensibilidade do método, uma vez que influencia a quantidade dos analitos que pode ser transferida para a solução utilizada nas medições. A fim de testar este efeito, a massa de amostra foi variada $(0,10$ a $1,2 \mathrm{~g})$ mantendo-se o volume de solução extratora constante em $25 \mathrm{~mL}$, volume empregado em todos os estudos anteriores. Os resultados obtidos estão mostrados na Figura 4.

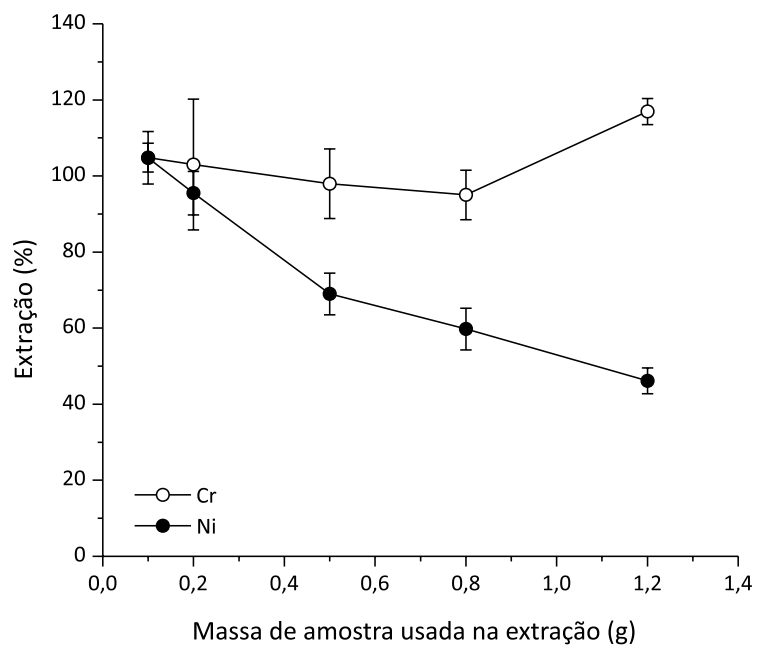

Figura 4. Influência da massa de amostra na extração de Cr e Ni empregando agitação magnética. Solução extratora $=\mathrm{HCl} \mathrm{2,0} \mathrm{mol} \mathrm{L}^{-1}$ e volume de solução extratora $=25 \mathrm{~mL}$

Para o Ni, não houve alteração significativa da eficiência de extração em toda a faixa estudada, indicando que este metal pode ser facilmente extraído das amostras pela ação do $\mathrm{HCl}$. Ao contrário, para o Cr, observa-se uma queda do percentual de extração quando a massa de amostra é superior a 0,20 g, pelo menos para a amostra composta utilizada nos estudos. Assim, com o intuito de desenvolver uma metodologia de extração que fosse compatível com a determinação dos dois metais estudados, uma massa de $0,20 \mathrm{~g}$ foi fixada para o método.

\section{Características analíticas do método desenvolvido}

Antes da determinação dos parâmetros analíticos da metodologia desenvolvida, foi realizado um estudo com o objetivo de verificar a ocorrência de interferências de matriz na determinação dos metais nos extratos obtidos na extração. Para este fim, para cada metal, curvas analíticas foram comparadas com curvas de adição padrão. Não foi observada diferença estatística ( $95 \%$ de confiança) entre as inclinações das retas obtidas (Tabela 3) para ambos os metais, indicando, como esperado, que as interferências de matriz estavam ausentes. Deste modo, foram utilizadas curvas analíticas preparadas em meio aquoso para quantificação dos metais nos extratos e, consequentemente, nas amostras.

A partir das curvas analíticas preparadas em meio de $\mathrm{HCl}$ 2,0 $\mathrm{mol} \mathrm{L}^{-1}$ (meio escolhido para a extração) foi possível calcular os limites de detecção $(3 \sigma / \mathrm{S})$ e de quantificação $(10 \sigma / \mathrm{S})$, onde

Tabela 3. Equações das curvas analíticas e das curvas de adição de padrão $(n=3)$

\begin{tabular}{|c|c|c|}
\hline Analito & Curva analítica & Curva de adição de padrão \\
\hline Cromo & $\begin{array}{c}\mathrm{A}=(0,0155 \pm 0,0003) \mathrm{C}_{\mathrm{A}}+(0,0038 \pm 0,0022) \\
\mathrm{r}^{2}=0,9995 \\
\text { Faixa de trabalho: } 0 \text { a } 30 \mu \mathrm{g} \mathrm{L}^{-1}\end{array}$ & $\begin{array}{c}\mathrm{A}=(0,0160 \pm 0,0004) \mathrm{C}_{\mathrm{A}}+(0,2760 \pm 0,0056) \\
\mathrm{r}^{2}=0,9998 \\
\text { Faixa de adição: } 0 \text { a } 10 \mu \mathrm{g} \mathrm{L} \mathrm{L}^{-1}\end{array}$ \\
\hline Níquel & $\begin{array}{c}\mathrm{A}=(0,0022 \pm 0,0008) \mathrm{C}_{\mathrm{A}}+(0,0035 \pm 0,0001) \\
\mathrm{r}^{2}=0,9994 \\
\text { Faixa de trabalho: } 0 \text { a } 30 \mu \mathrm{g} \mathrm{L}^{-1}\end{array}$ & $\begin{array}{c}\mathrm{A}=(0,0024 \pm 0,0003) \mathrm{C}_{\mathrm{A}}+(0,0174 \pm 0,0025) \\
\mathrm{r}^{2}=0,9995 \\
\text { Faixa de adição: } 0 \text { a } 15 \mu \mathrm{g} \mathrm{L}{ }^{-1}\end{array}$ \\
\hline
\end{tabular}


Tabela 4. Resultados obtidos na determinação de Cr e Ni empregando a extração assistida por agitação magnética e a dissolução total assistida por radiação micro-ondas. As concentrações $\left(\mu \mathrm{g} \mathrm{g}^{-1}\right)$ dos analitos são expressas como a média \pm desvio padrão $(\mathrm{n}=3)$

\begin{tabular}{|c|c|c|c|c|c|}
\hline \multirow{2}{*}{ Amostra } & \multirow{2}{*}{ Tipo* } & \multicolumn{2}{|c|}{ Cromo } & \multicolumn{2}{|c|}{ Níquel } \\
\hline & & Agitação magnética & Dissolução total & Agitação magnética & Dissolução total \\
\hline $\mathrm{S}_{1}$ & Ciprofloxacino FF & $5,58 \pm 0,40$ & $5,66 \pm 0,61$ & $1,18 \pm 0,12$ & $1,26 \pm 0,21$ \\
\hline $\mathrm{S}_{2}$ & Ciprofloxacino MP & $0,79 \pm 0,06$ & $0,90 \pm 0,05$ & $0,50 \pm 0,04$ & $0,49 \pm 0,07$ \\
\hline $\mathrm{S}_{3}$ & Cefalexina FF & $1,50 \pm 0,02$ & $1,67 \pm 0,02$ & $1,55 \pm 0,07$ & $1,70 \pm 0,28$ \\
\hline $\mathrm{S}_{4}$ & Cefalexina MP & $1,42 \pm 0,14$ & $1,60 \pm 0,21$ & $0,51 \pm 0,01$ & $0,57 \pm 0,12$ \\
\hline $\mathrm{S}_{5}$ & Cefalexina FF & $0,96 \pm 0,06$ & $1,01 \pm 0,07$ & $0,94 \pm 0,05$ & $0,91 \pm 0,09$ \\
\hline
\end{tabular}

* Os índices FF e MP representam formulação farmacêutica e matéria-prima, respectivamente.

$\sigma$ representa o ruído instrumental e $\mathrm{S}$ a sensibilidade da curva analítica, representada pela sua inclinação. ${ }^{15} \mathrm{O}$ ruído instrumental foi estimado a partir de 10 medições da solução branco $\left(\mathrm{HCl} 2,0 \mathrm{~mol} \mathrm{~L}^{-1}\right)$. Para o Cr, os limites de detecção e de quantificação em solução (no extrato) foram 0,19 e $0,64 \mu \mathrm{g} \mathrm{L}{ }^{-1}$, respectivamente. Já para o Ni, os limites de detecção e de quantificação em solução (no extrato) foram 0,64 e $2,1 \mu \mathrm{g} \mathrm{L}^{-1}$, respectivamente. Levando-se em consideração o processo de extração $(0,20 \mathrm{~g}$ de amostra com $25 \mathrm{~mL}$ de solução extratora) é possível calcular os limites de detecção e quantificação na própria amostra sólida. Neste caso, os limites de detecção foram 24 e $80 \mathrm{ng} \mathrm{g}^{-1}$ para $\mathrm{Cr}$ e Ni, respectivamente, e os limites de quantificação foram 80 e $262 \mathrm{ng} \mathrm{g}^{-1}$ para $\mathrm{Cr}$ e $\mathrm{Ni}$, respectivamente.

A precisão do método também foi avaliada. Neste caso 10 alíquotas da amostra $\mathrm{S}_{5}$ foram tomadas, de modo independente, e foram determinadas as concentrações de $\mathrm{Cr}$ e Ni. O desvio padrão relativo obtido foi igual a $6,7 \%$ para o $\mathrm{Cr}$ e $9,6 \%$ para o $\mathrm{Ni}$.

\section{APLICAÇÃO DA METODOLOGIA DESENVOLVIDA EM AMOSTRAS REAIS}

A fim de avaliar a exatidão da metodologia desenvolvida, duas estratégias foram testadas. Primeiramente, foram realizados ensaios de recuperação pela adição de quantidades conhecidas de $\mathrm{Cr}$ e Ni às amostras, antes do processo de extração, em dois diferentes níveis de concentração - 6,0 e $12 \mu \mathrm{g} \mathrm{L}^{-1}$ para $\mathrm{Cr}$ e 5,0 e $10 \mu \mathrm{g} \mathrm{L}^{-1}$ para Ni. Os percentuais de recuperação variaram entre 84 e $102 \%$ para o $\mathrm{Cr}$ e entre 92 e $96 \%$ para o Ni.

Os resultados obtidos através da extração assistida por agitação magnética foram também comparados com os resultados obtidos através da dissolução total da amostra assistida por radiação micro-ondas focalizada (Tabela 4). A comparação entre os valores obtidos não apresentou diferença estatística significativa (teste t pareado, $95 \%$ de confiança).

As amostras analisadas foram provenientes de formulações farmacêuticas (FF) e matérias-primas utilizadas para manipulação de fórmulas magistrais (MP) comercializadas no Brasil. Como a matéria-prima não foi a mesma utilizada na preparação das formulações farmacêuticas, não foi possível estabelecer uma relação entre a contaminação das formulações farmacêuticas com metais a partir da sua introdução via matérias-primas. Entretanto, é importante ressaltar que o limite máximo aceito pela Farmacopeia Brasileira, $5^{\text {a }}$ ed., para $\mathrm{Cr}$ e $\mathrm{Ni}$ em produtos farmacêuticos é de $25 \mu \mathrm{g} \mathrm{g}^{-1}$ e que as concentrações de $\mathrm{Cr}$ e $\mathrm{Ni}$ encontradas nas cinco amostras analisadas foram sempre inferiores a este limite.

\section{CONCLUSÕES}

Os resultados obtidos demonstraram que o uso de métodos de extração pode ser uma excelente alternativa aos métodos convencionais de tratamento de amostras sólidas para a preparação destas, visando a determinação de metais por GFAAS. Entre os métodos de extração estudados, o emprego da agitação magnética se mostrou mais eficiente do que a extração empregando banho de ultrassom e do que a extração assistida por radiação micro-ondas focalizada.

Os maiores benefícios observados quando do uso do método de extração proposto, em comparação com os métodos convencionais, foram a utilização de soluções diluídas de ácidos, que resultou em valores mais baixos dos brancos analíticos, e a necessidade de uso de equipamentos de baixo custo e fácil acesso.

O método desenvolvido apresentou limites de quantificação bem inferiores aos limites máximos permitidos de $\mathrm{Cr}$ e $\mathrm{Ni}$ pela Farmacopeia Brasileira em produtos farmacêuticos podendo, assim, ser facilmente utilizado para o controle de qualidade de medicamentos sólidos. Sua aplicabilidade pode ser estendida para outros princípios ativos e para outros analitos.

\section{MATERIAL SUPLEMENTAR}

Está disponível em http://quimicanova.sbq.org.br com acesso livre ao arquivo PDF, composto pelas Figuras $1 \mathrm{~S}$ a $4 \mathrm{~S}$.

\section{AGRADECIMENTOS}

Ao CNPq (Conselho Nacional de Desenvolvimento Científico e Tecnológico), à CAPES (Coordenação de Aperfeiçoamento de Pessoal de Nível Superior) e à FAPERJ (Fundação Carlos Chagas Filho de Apoio à Pesquisa do Estado do Rio de Janeiro) pelas bolsas de estudo e pesquisa concedidas e pelos auxílios financeiros que tornaram possível a realização deste estudo.

\section{REFERÊNCIAS}

1. Farmacopeia Brasileira; 2010, $5^{\mathrm{a}}$ ed., Parte I, p. 171.

2. Gil, E. S.; Controle Físico-Químico de Qualidade de Medicamentos, $3^{\mathrm{a}}$ ed., LMC-Pharmabooks Ed.: São Paulo, 2007.

3. Quintaes, K. D.; Farfan, J. A.; Tomazini, F. M.; Morgano, M. A.; Mantovani, D. M. B.; Ciênc. Tecnol. Aliment. 2004, 24, 397.

4. The United States Pharmacopeia; The National Formulary - USP 32; NF- 27, 2009.

5. Lewen, N.; J. Pharm. Biomed. Anal. 2011, 55, 653.

6. Filgueiras, A. V.; Capelo, J. L.; Lavilla, I.; Bendicho, C.; Talanta 2000, $53,433$.

7. Flores, E. M. M.; Paniz, J. N. G.; Martins, A. F. M.; Dressler, V. L.; Muller, E. I.; Costa, A. B. C.; Spectrochim. Acta, Part B 2002, 57, 2187.

8. Bolzan, R. C.; Moraes, D. P.; de Mattos, J. C. P.; Dressler, V. L.; Flores, E. M. M.; J. Braz. Chem. Soc. 2010, 21, 686.

9. Resano, M.; Bricieno, J.; Aramendia, M.; Belarra, M. A.; Anal. Chim. Acta 2007, 582, 214. 
10. Heinemann, G.; Vogt, W.; Biol. Trac. Elem. Res. 2000, 75, 227.

11. Zachariadis, G. A.; Sahanidou, E.; Cent. Eur. J. Chem. 2011, 9, 213.

12. Niemeza, M.; Kola, H.; Eilola, K.; Peramaki, P.; J. Pharm. Biomed. Anal. 2004, 35, 433.

13. Ponce de León, C. A.; Bayón, M. M.; Caruso, J. A.; Anal. Bioanal. Chem. 2002, 374, 230.

14. Oliveira, E.; J. Braz. Chem. Soc. 2003, 14, 174.

15. Valcárcel, M.; Principios de Quimica Analitica, Springer-Iberica: Barcelona, 1999.

16. Krug, F. J.; Métodos de preparo de amostras: fundamentos sobre métodos de preparo de amostras orgânicas e inorgânicas para análise elementar, 1ํㅡㄹ. ed., Edição do autor: Piracicaba, 2008.
17. Hoenig, M.; de Kersabiec, A.-M.; Spectrochim. Acta, Part B 1996, 51, 1297.

18. Antes, F. G.; Santos, M. F. P.; Guimarães, R. C. L.; Paniz, J. N. G.; Flores, E. M. M.; Dressler, V. L.; Anal. Methods 2011, 288, 3.

19. Flores, E. M. M.; Barin, J. S.; Paniz, J. N. G.; Medeiros, J. A.; Knapp, G.; Anal. Chem. 2004, 76, 3525.

20. Soriano, S.; Pereira-Netto, A. D.; Cassella, R. J.; J. Pharm. Biomed. Anal. 2007, 43, 304.

21. Soriano, S.; Pereira-Netto, A. D.; Cassella, R. J.; Anal. Bioanal. Chem. 2007, 387, 1113. 


\section{AVALIAÇÃO DE MÉTODOS DE EXTRAÇÃO PARA A DETERMINAÇÃO DE CROMO E NÍQUEL EM FORMULAÇÕES FARMACÊUTICAS E EM MATÉRIAS-PRIMAS USADAS NA FABRICAÇÃO DE MEDICAMENTOS À BASE DE CEFALEXINA E CIPROFLOXACINO}

\section{Carlos Eduardo R. de Paula}

Departamento de Química Analítica, Universidade Federal Fluminense, Outeiro de São João Batista, s/n, 24020-141 Niterói - RJ / Departamento de Química, Universidade Federal Rural do Rio de Janeiro, BR-465, km 7, 23890-000 Seropédica - RJ, Brasil Daniel M. Brum, Luiz Fernando S. Caldas e Ricardo J. Cassella*

Departamento de Química Analítica, Universidade Federal Fluminense, Outeiro de São João Batista, s/n, 24020-141 Niterói - RJ, Brasil
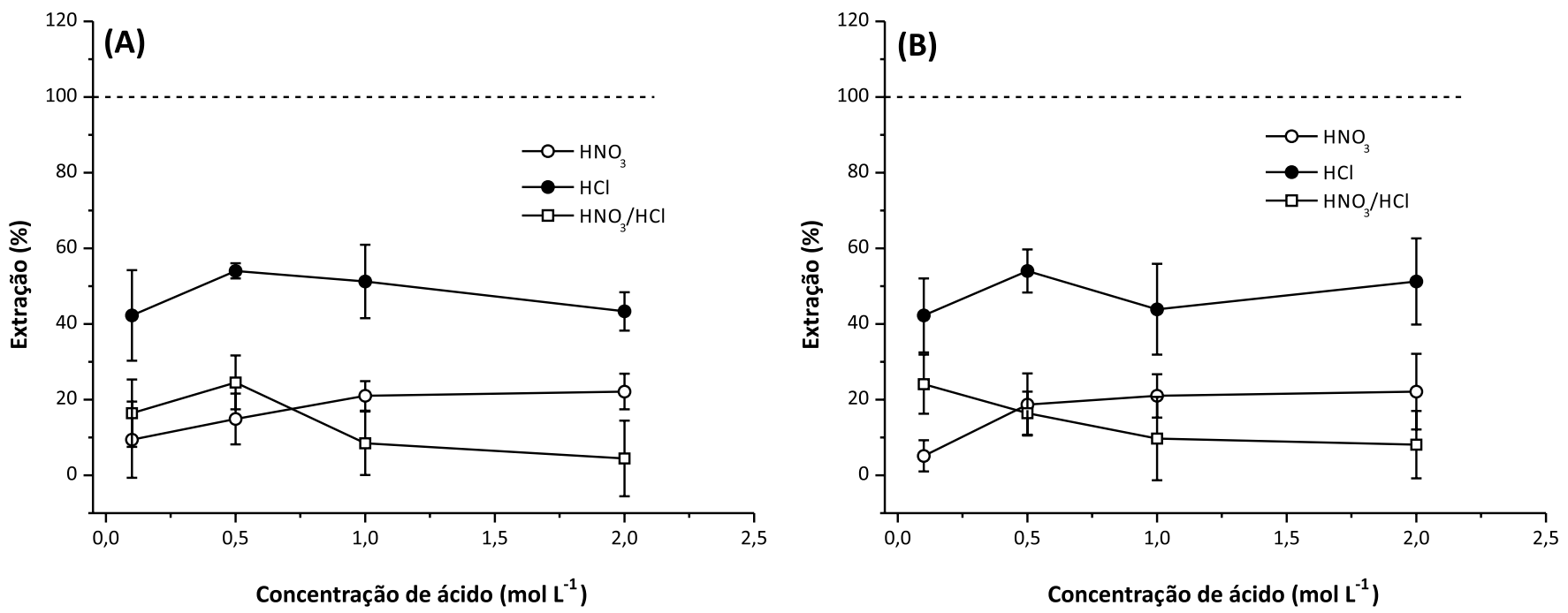

Figura 1S. Influência da natureza e da concentração do ácido na extração de $\mathrm{Cr}(\mathrm{A})$ e $\mathrm{Ni}(\mathrm{B})$ por ultrassom. Detalhes sobre os tempos de extração podem ser encontrados no texto
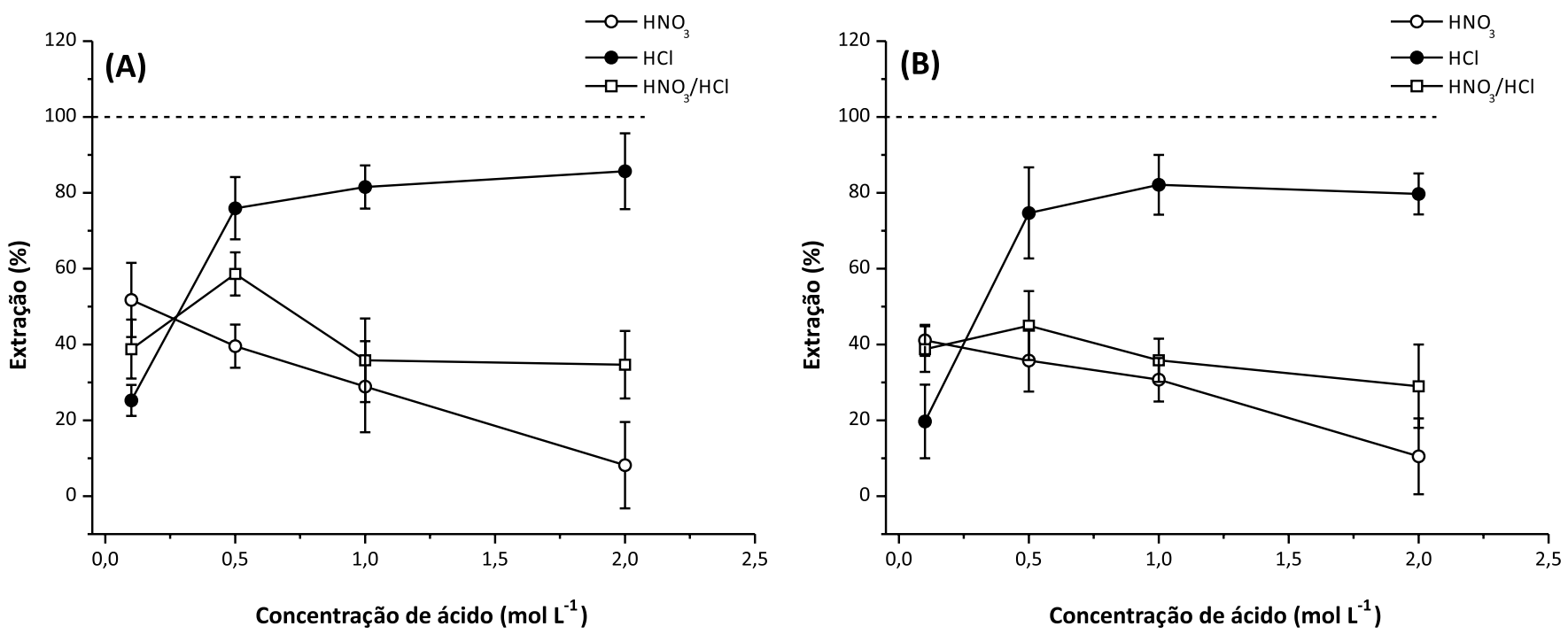

Figura 2S. Influência da natureza e da concentração do ácido na extração de Cr (A) e Ni (B) por radiação micro-ondas focalizada. Detalhes sobre a concentração de ácido (ou mistura) na solução extratora podem ser encontrados no texto

*e-mail: cassella@vm.uff.br 

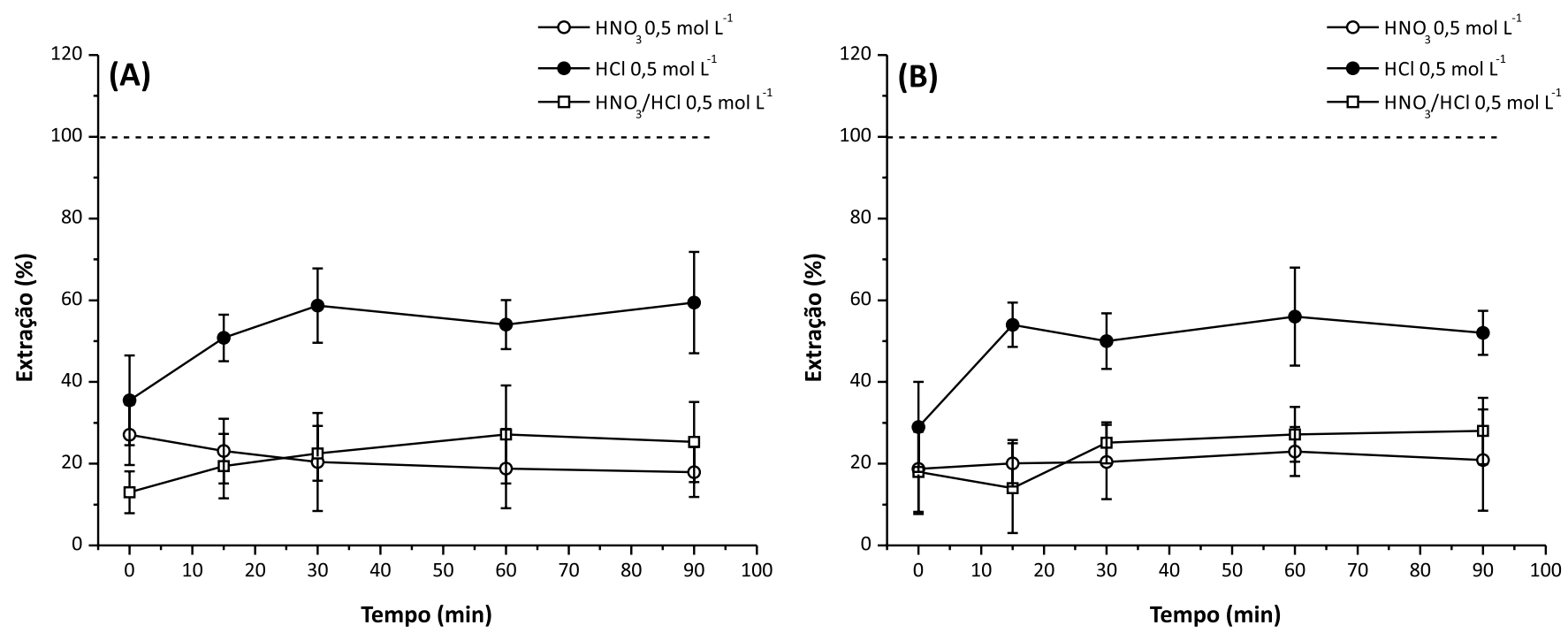

Figura 3S. Influência do tempo na extração de $\mathrm{Cr}(\mathrm{A})$ e $\mathrm{Ni}(B)$ por ultrassom. Detalhes sobre os tempos de extração podem ser encontrados no texto
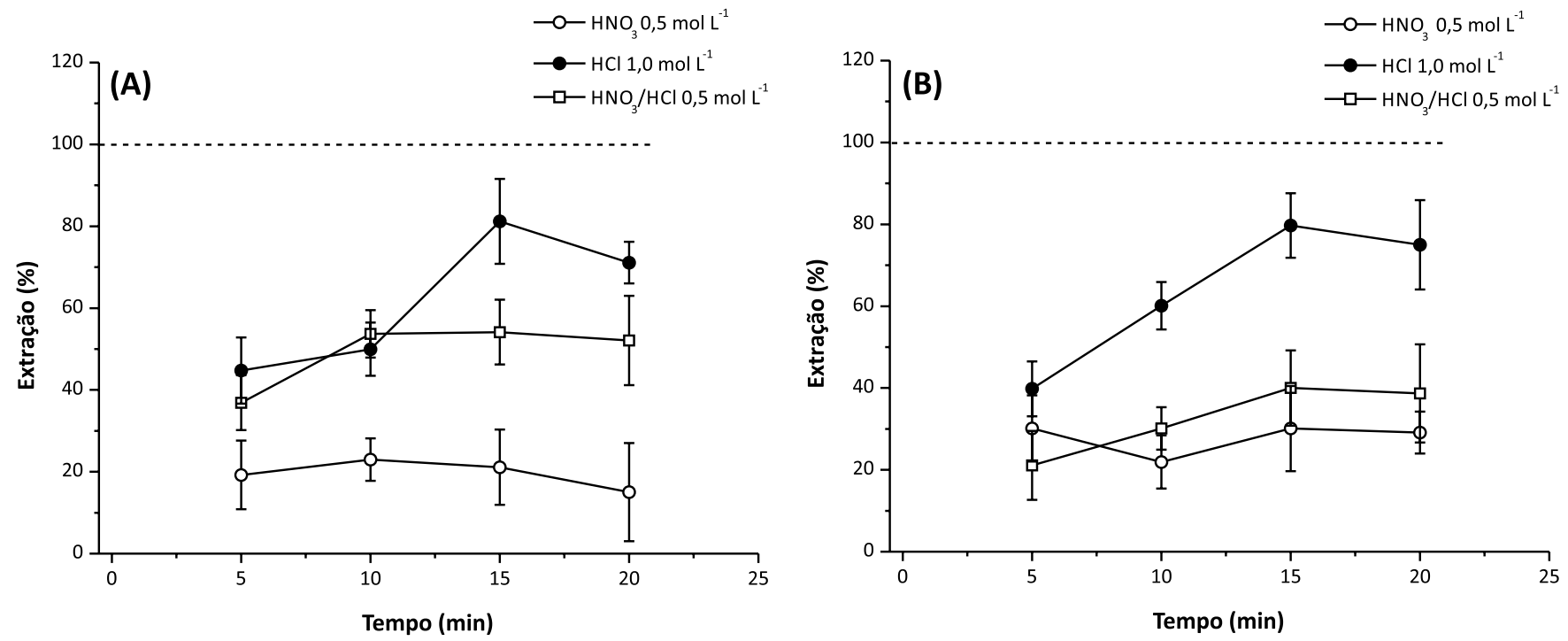

Figura 4S. Influência do tempo na extração de $\operatorname{Cr}(A)$ e Ni (B) por radiação micro-ondas focalizada. Detalhes sobre a concentração de ácido (ou mistura) na solução extratora podem ser encontrados no texto 